\title{
VIII. Über Chalkopyrit von Arakawa, Provinz Ugo in Japan, und über die Structur des Chalkopyrits im allgemeinen.
}

\author{
Von \\ J. Beckenkamp in Würzburg. \\ (Hierzu Tafel I.)

\section{Chalkopyrit von Arakawa.}

\section{Literatur.}

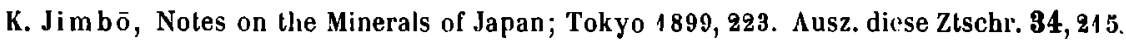
'T. Wada, Minerals of Japan; Tokyo 1904, 32-34. Ausz. am Schlusse dieses Heftes. K. Jimbō, A Complex Twin of Chalkopyrite from Arakawa, Ugo Province. Beiträge zur Mineralogie von Japan, herausgegeben von T. Wa da 1906, 70-71.

In der zuerst genannten Mitteilung erwähnt Jimbō einen merkwürdigen, dreieckigen keilförmigen Krystall von Arakawa aus der Sammlung von T. Wada.

T. Wada berichtet 1904, daß gut krystallisierter Chalkopyrit in Japan fast ausschließlich auf den Kupfererzgängen in Gebieten mit jüngeren vulkanischen Gesteinen gefunden würde. Nach der Krystallform unterscheidet er fünf Typen ${ }^{1}$ ):

1) Einfache bisphenoidische Krystalle mit dem positiven Sphenoide $p\{111\}$ (Taf. I, Fig. 1).

2) Combination von $y\{313\}$ mit $X\{331\}$ (Fig. 2).

3) Combination von $x\{212\}, t\{221\}$ und $p\{111\}$; Drillinge nach (111) (Fig. 3).

4) Dreieckige Krystalle einer Combination von $m\{110\}, \chi\{212\}$ und $p\{111\}$ (Fig. 4).

5) Nadelförmige Krystalle mit denselben Formen wie 4) (Fig. 5).

1) Die Figuren 2-.j sind der Mlitteilung von Wad a entlehnt, die Figuren 6-8 der von Jimbo. 
Nach Wada ist die erste Form allgemein sehr häufig, dagegen die vierte und fünfte auf Arakawa beschränkt. Als Begleitmineralien werden für Arakawa kleine Quarzkrystalle und Pyrit angegeben. Die größten Krystalle des vierten Typus erreichen einen Durchmesser von $60 \mathrm{~mm}$; die des fünften Typus eine Länge von $35 \mathrm{~mm}$ bei einer Breite von nur $\mathbf{2} \mathbf{m m}$. Chalkopyrit und Quarz bilden Pseudomorphosen nach Baryt; auch kommen Überzüge von Baryt auf Chalkopyrit häufig vor.

Jimb $\overline{0}$ fand auch auf einer Stufe von Arakawa neben dem vierten Typus mehrere Drillinge nach dem dritten Typus. Die Orientierung derselben wird durch die Streifung erleichtert; $m$ und $p$ sind nach Jimb $\overrightarrow{0}$ horizontal gestreift, die Streifung auf $\chi$ wird von ihm nicht näher charakterisiert, sondern nur erwähnt, daß sie ähnlich sei, wie sie Dana in seiner Fig. 10, S. 81 (in System of Mineralogy 1892) angebe (vgl. Fig. 6 und 7).

Die Fig. 7 und 8 sollen die Drillingsbildung erläutern; es wird angenommen, daß man das mittlere Individuum, welches einen Keil zwischen den beiden anderen bildet, in der Richtung der verticalen Axe senkrecht zur Basis betrachte.

Jimbō gibt ferner an, daß er in Wakabayashis Sammlung eine dreieckige Platte von Arakawa gesehen habe, deren eine Oberfläche fast eben und glatt sei, die andere dagegen kleine Flächen an der Seite zeige; ferner eine sehr kleine rechteckige Platte von demselben Fundorte mit ähnlicher Oberfläche, von welchen aber die eine Furchen parallel zu den kürzeren Seiten des Rechteckes habe; endlich eine dreieckige Platte von dort mit einem langen prismatischen Individuum, von welchem er es fraglich hinstellt, ob es in Zwillingsstellung mit der Platte stehe.

\section{Beobachtungen.}

l)as Ilaterial der nachstehenden Untersuchungen stammt aus der Sammlung Wadas; es wurde von ihm der Münchener Staatssammlung geschenkt und mir von Prof. v. Groth zur Untersuchung überlassen. Die drei ersten Typen Wadas sind unter dem mir vorliegenden Vaterial nicht vertreten, dagegen in reichlicher Anzahl Vertreter des vierten und fünften Typus, ferner einige dünnplattige Formen, ähnlich den von Jimbō erwähnten, und eine Verwachsung von Chalkopyrit mit Chalkolyyrit und Pyrit. Die Mehrzahl der Krystalle, besonders die nadelförmigen, sind von einer dunklen Oxydschicht überzogen. Außerdem sind auf einer Fläche eines größeren Krystalles vom vierten Typus Kryställchen von Pyrit und Quarz unregelmäßig aufgewachsen.

Vierter Typus (Fig. 9 und 10).

Von den dreiseitigen flachen Formen lagen mir sechs größere Krystalle mil 17 bis $35 \mathrm{~mm}$ langer Kante und 3 bis $8 \mathrm{~mm}$ Dicke und zwei 
kleinere mit 6 und $7 \mathrm{~mm}$ Kantenlänge vor. Die beiden letzteren können übrigens als Übergang zum fünften Typus gelten.

Nach der gewöhnlichen tetragonalen Auffassung kommen folgende Formen vor: $x\{1 T T\}, p\{1 \mid 1\}, p^{\prime}\{1 T 1\}, m\{1 T 0\}, e\{101\}$ ?, $c\{001\}$; ferner $n\{112\}$ viermal, $n^{\prime}\{1 T 2\}$ zweimal, $\gamma^{\prime}\{1 T 5\}$ einmal, " $\{025\}$ zweimal.

Das positive Bisphenoid zerfällt in zwei völlig verschiedene Hälften: die beiden unteren Flächen $\pi(1 T T)$ und $(T / T)$ und die oberen $p(1 \mid 1)$ und (TT1). Die beiden ersteren stellen stets eine nach ihrer Schnittkante langgestreckte messerartige Form dar, welche für das Vorkommen von Arakawa äußerst bezeichnend ist. Die Form $p$ dagegen ist verhältnismäßig. kleiner entwickelt, liegt in den äußersten Ecken über jener langen Kante und wechselt meist mit den darüber liegenden, vorhin mit $e$ bezeichneten Flächen $a b$.

Sowohl $\tau$ als $p$ lassen hïufig eine Streifung erkennen, welche ihren Combinationskanten parallel lüuft, indem von einer Stelle, gewissermaßen von einer höheren mit der Hauptfläche $x$ (bezïglich $p$ ) genau parallelen Terrasse aus ein stufenweiser Abfall nach der Hauptläche erfolgt (vergl. Fig. 9. Gewöhnlich stehen die so gebildeten erhöhten Dreiecke einzeln; bei einem der grüßeren Krystalle liegen auf $\tau$ die oberen Enden einer größeren $\Lambda$ nzahl derartiger Dreiecke in einer langen zur Kante (1TT): (T1T) parallelen Reihe, während die Grundlinien dieser Dreiecke alle in dieser Kante liegen.

$\Delta u f$ beiden Flächen $\boldsymbol{t}$ finden sich an der oberen Grenze gegen $m$ bezüglich $p^{\prime}$ zuweilen auch ein einzelner, oder doch nur wenige Streifen parallel zur Kante $x: m$, welche ebenfalls einen Terrassenabfall und zwar hier von $\pi$ gegen $m$ erkennen Jassen.

Der grüßere Teil der Fläche $z$ ist in der Regel glatl und gibt gute Bilder, während von $p$ meist nur ein kleiner Teil glatt, der größer'e Teil durch alternierende Ausbildung mit $e$ gestreift ist.

Die Messung des Winkels $x: x=(1 T T):(T \backslash T)$ ergab im Mittel 108046', daraus folgt $c: x=(00 \bar{T}):(1 \bar{T} \bar{T})=54023^{\prime}$.

Das negative Bisphenoid $p^{\prime}$ trilt nur mit den beiden oberen Flächen (1T1) und (T11) auf, und ist nur bei den beiden kleineren Krystallen dieses Typus verhältnismäßig groß entwickelt. Eine Streifung wurde auf $p^{\prime}$ nur in geringerem Maße beobachtet; vereinzelt bildet $m$ eine oder mehrere schmale Stufen auf $p^{\prime}$.

Das Prisma $m$ wurde ebenso, wie $p^{\prime}$, immer nur mit zwei Flächen $(1 \bar{T} 0)$ und $(\overline{1} 10)$ beobachtet; $m$ ist stets stark gestreift durch abwechselnde Ausbildung mit $\pi$. IDie Winkelwerte sind deshalb sehr schwankend, und die Reflexbilder liegen immer näher an $x$ als an $p^{\prime}$. \%wischen $m$ und $x$ erscheint meist ein ganzes Band von Reflexbildern, aber es war nicht mög- 
lich, bestimmte Bilder, welche etwa in derselben Lage bei mehreren Krystallen wiederkehrten, zur Bestimmung von bestimmten spitzen Sphenoïden $\mathrm{zwischen}(1 \bar{T} 0)$ und $(1 \bar{T} \bar{T})$ herauszufinden 1$)$.

Die Grundpyramide $\mathrm{z}$ weiter Art $e\{101\}$ tritt nur mit den vier oberen Flächen auf, ist niemals glänzend und meist stark gestreift in der Richtung der Zonenaxe (111):(1T1) durch alternierende Ausbildung mit $p$ (111). Auch die Flächen von $e$ liefern in der Regel eine ganze Reihe von Bildern $z$ wischen (101) und (111); ist nur ein Bild sichtbar, so liegt dies immer näher bei $p$ als bei $p^{\prime}$. Ebensowenig wie bei $m$ lassen sich unter diesen Streifen Bilder von bestimmter Lage herauslesen; es wurde deshall das Zeichen $e$ als das der Grenzfläche der Reihe gewählt. Wada gibt in Fig. 3 statt dessen die Form $\chi\{212\}$ an, indessen bemerkt auch Jimbō, daß nach Dana diese Form bei anderen Fundorten unbestimmt sei. Penfield schreibt mit Bezug auf diese Form in seiner Mitteilung über Kupferkieskrystalle aus den French Creek-Eisengruben in Pensylvanien ${ }^{2)}$ : »Das Skalenoëder $\%$ ist stark gestreift; Messungen mit dem Anlegegoniometer ergaben für die längere und kürzere Polkante in einer Anzahl von Fällen Winkel von $40^{\circ}$ und $90^{\circ}$, Werte, welche sich der Form $\{122\}$ nähern, deren Polkanten 381.0 und 901.0 messen würden." "Von $p(111)$ ausgehend folgt in der Zone $p(111): p(1 \bar{T} 1)$ ein ununterbrochenes Band von Reflexen bis ungefïhr $35^{\circ}$, d. i. von (111) bis zu einer Pyramide zweiter Ordnung e(101), nach welcher kein Reflex mehr erhalten wurde bis zu $p^{\prime}$. Es ist also wahrscheinlich, daß kein bestimmtes Sphenoid in dieser Zone liegt, und daß $\%$, welches zu $\{122\}$ genommen wurde, durch die oscillatorische Combination von $e\{101\}$ und $p\{111\}$ hervorgebracht wird. " Meine Beobachtungen an den Krystallen von Arakawa bestätigen diese Annahme.

Die Basis $c\{001\}$ wurde nur bei zwei der größeren, aber bei beiden kleineren Krystallen beobachtet; sie tritt nur mit einer, und zwar der oberen Fläche (001) auf. Diese ist meist glatt, nur an einem der kleineren Krystalle ist rechts und links durch je einmaliges Einsetzen von $p$ ein $\mathbf{A b}-$ fallen von einer höheren mittler'en 'T'errasse zu einer niedrigeren äußeren Terrasse zu erkennen.

Die übrigen Formen sind nur von untergeordneter' Bedeutung: $n\{112\}$ bildet zuweilen eine schmale gerade Abstumpfung der Kanten

1) Die Erscheinung, da $\beta$ hier nur der in den betreffenden Flachen liegenden Zonenaxe, nicht aber der Normalen der in Frage kommenden einzelnen Flächen eine constante Lage zukommt, halte ich für einen Beweis für die von mir vertretene Ansicht, daß die Krystallläche mechanisch nicht durch die zu ihrer Lage normale, sondern durch wenigstens zwei in der Fläche liegende tangentiale hrüfte definiert ist. Vgl. diese Zeitschr. 36, 111.

2) Diese Zeitschr. 1891, 18, 513. 
$e: e=(101):(011)$, während $n^{\prime}\{1 \overline{1} 2\}$ eine schmale Abstumpfung der Kanten $p^{\prime}: c=(1 \pi 1):(001)$, besonders der kleineren Krystalle darstellt. Noch seltener und ebenfalls zwischen (1T1) und (001) gelegen ist $\gamma^{\prime}\{1 \bar{T} 5\}$.

Die Form " $\{025\}$ scheint beim Kupferkies bis jetzt nicht beobachtet zu sein; sie wurde mil den beiden Flächen $(025)$ und $(0 \overline{2} 5)$ zu beiden Seiten von $c$ zwischen $c$ und $e$ beobachtet. Der beobachtete Winkel (025): $(0 \overline{2} 5)$ ist $43^{0} 10^{\prime}$, der berechnete Wert $43^{\circ} 2^{\prime}$.

Penfield bemerkt bezüglich der Krystalle aus den French CreekEisengruben: "Bei der Messung von $p(\bar{T} T 1)$ über die Basis zu $p(111)$ ergaben sich keine distincten Reflexe mit Ausnahme von $p$ und $c$, und bei weiterer Drehung folgte unmittelbar ein ununterbrochenes Band von Reflexen ohne irgendwelche Unterbrechung der hervortretenden Teile zwischen $p$ und $m(110)$. "Diese Streifung zwischen (111) und (110) entspricht der vorhin von mir beschriebenen Beobachtung an den Krystallen von Arakawa; dagegen ließ das japanische Vorkommen zwischen (111) und (001) die, wenn auch nicht gerade häufigen Formen $\{112\}$ und $\{115\}$ erkennen und zwischen (1T1) und (001) die Form $\left\{1 T_{2}\right\}$.

Fünfter Typus (Fig. 11).

Der Charakter der Krystalle des fünften Typus wird wesentlich durch deren Verlängerung in der Richtung der unteren Kante $\pi: \pi$ bedingt. Das Verhältnis der Länge zur Dicke kann derart zunehmen, daß sehr feine lange Nadeln entstehen. Es lagen mir 37 Krystalle dieser Art vor, deren längster bei ca. $48 \mathrm{~mm}$ Länge einen Durchmesser von $\frac{1}{2} \mathrm{~mm}$ besitzt.

Die Flächenbildung und Streifung ist im wesentlichen dieselbe wie beim vorigen Typus, nur fehlt bei den langgestreckten Krystallen die Basis wohl kaum; aber auch hier tritt sie immer nur mit einer einzigen, der oberen Fläche auf. Das Einsetzen der Flächen (111) bezüglich (TTT) ist hier häufiger, und deshalb (001) lü̈uliger nach der betreffenden Combinationskante gestreift.

Mitunter ragt mitten über einer Basislliche eines langgestreckten Krystalles eine kleine dreiseilige Erhebung, umgrenzt von den vier oberen Flächen von $e$ und den beiden Flächen von $m$; man kann ein derartiges Gebilde gewissermaßen als cine Vereinigung je eines Individuums des vierten und fünften Typus in paralleler Orientiejung ansehen. Oflenbar gehört hierher auch die oben (S. 44) erwähnte, von Jimbö angegebene Verbindung der dreiseitigen Platte mit dem prismalischen Krystalle.

Nichl selten wiederholen sich die dreiseitig plattenfürmigen Auswüchse auf der gleichen Basis und dann erscheint letztere wie mit Zähnen besetzt.

Als kleine dreieckige, die Ecke zwischen (001): 101): (011) abstumpfende Flïche fand ich einmal $x\{113\}$.

Bei weitem die Mehrzahl der Krystalle war an einem Ende abgebrochen. 
Bei den wenigen beiderseils ausgebildeten schienen zuweilen an dem einen Kopfe fast ausschließlich die beiden Flächen $e$ mit ganz untergeordnetem $p$, am anderen Kopfe neben $e$ auch $p$ größer entwickelt und auflallender mit $e$ alternierend. Indessen ist die Anzahl der hierfür in Betracht kommenden Krystalle zu gering, als daß daraus eine Gesetzmäßigkeit abgeleitet werden könnte.

Eine dreiseitige düntafelige Platte (Fig. 12).

Die mir vorliegende dünntafelige dreiseitige Plalte besitzt etwa $7 \mathrm{~mm}$ Seitenlänge und $1-2 \mathrm{~mm}$ Dicke. Die untere dreiseitige Fläche $\pi(\bar{T} \mid \bar{T})$ ist vollständig glatt, die obere $p^{\prime}(1 T 1)$ von einigen Streifen durchzogen, welche durch das Einsetzen von $m$ hervorgebracht werden.

Außer den größeren Randflächen $e(101)$ und (0T/), sowie $m(1 T 0)$ wurde an der unteren rechten Ecke als sehr schmale Fläche $e(01 \overline{1})$ beobachtet. Die Flächen $e$ und $m$ wurden durch Schimmermessung bestimmt. Wie gewöhnlich, so entfernen sich auch hier beide Flächen um $3^{0}$ bis $4^{0}$ weiter von $p^{\prime}$, als es der normalen Lage von $e$ und $m$ entsprechen würde. Die nach der Sphenoidfläche tafelförmige Ausbildung dieser Platte ist auffallend; für die Annahme, daß die Tafelform mit einer Zwillingsbildung nach (111) in Zusammenhang stehe, liefern die vorhandenen Flächen keinen Beweis.

\section{Zwei rechteckige dünntafelige Platten.}

Die kleinere der beiden rechteckigen Platten hat eine Fläche von $\tilde{\mathrm{s}} \times 6 \mathrm{~mm}$ und ist beinahe papicrdünn. Auf der einen Tafelseite ist sie von zahlreichen kleinen Kupferkieskryställchen besetzt, welche mehr oder weniger den vorhergehenden 'Typen entsprechen. Außerdem laufen über beide 'Tafelseilen leistenfürınige Erhühungen, welche von $c, p$ und $e$ begrenzt sind; auf der einen Seite bewirken diese Leisten eine Streifung parallel zur längeren Seite der Platte, auf der mit den kleinen Kryställchen besetzten Seite dagegen parallel zu den kürzeren Seiten. Als Randllächen treten nur in Form sehr schmaler Zuschärfungen $p$ und $p^{\prime}$ auf.

Die größere Platle mißt $6 \times \mathbf{8} \mathrm{mm}$, aber eine der vier Ecken ist abgebrochen; die kleinen parasilischen Krystïllchen fehlen. Randllïchen und Streifung parallel $(001):(111)$ entsprechen dem vorigen; nur tritt auch $m$ wenigstens mit einer Fläche auf. Mitten über $m$ verläuft eine mit der lupe, deutlicher mit dem Mikroskope erkennbare Naht parallel der Schnittkante $m: c$.

Diese beiden rechteckigen, nach (001) tafelförmigen Krystalle unterscheiden sich sehr wesentlich von allen vorher beschriebenen dadurch, daß die Basis mit beiden und die Sphenoide mit je vier gleichartigen Flächen auftreten. 


\section{Verwachsung von Chalkopyrit mit Chalkopyrit und Pyrit}

(Fig. 13 und 14).

Unter den mir vorliegenden Krystallen befand sich einer, welcher den von Wada beschriebenen Jrillingen ähnlich ist, andererseits aber auch bedeutende Abweichungen von diesen zeigt. Die längste Dimension dieses Krystalles beträgt etwa $15 \mathrm{~mm}$, die mittlere $12 \mathrm{~mm}$, die kürzeste $6 \mathrm{~mm}$. Zwei größere Individuen 1 und II sind so mit einander verbunden, daß ihre beiden scharfen Kanten $\pi: \pi$ genau zusammenfallen, und daß die Normalen der diesen Kanten gegenüberliegenden Basisllichen $c_{\mathrm{I}}$ und $c_{\mathrm{II}}$ cinen Winkel von $95^{0} 56^{\prime}$ mit einander bilden. Bei den von Wada beschriebenen Drillingen beträgt der entsprechende Winkel $2 \times 71040^{\prime}=$ $143^{\circ} 20^{\prime}$.

Nimmt man an, beide Individuen seien spiegelbildlich zu einander in bezug auf (223), dann ist jener Winkel 940 14'. Die beiden Individuen entsprechen im einzelnen bezüglich Streifung und Habitus dem vierten Typus. Fig. 14 stellt einen Schnitt senkrecht zur gemeinsamen Zwillingskante dar. Die Fläche $\psi$ ist cine zu $m$ vicinale lläche, welche den früher besprochenen entspricht. Die Messung der Polkante von $e$ ergab 550 15', während der entsprechende Wert für o theoretisch $59030^{\prime}$ beträgt. Der Wert $55^{\circ} 15^{\prime}$ würde zu einer form $\{15.1 .15\}$ führen, für welche die Berechnung $550 z^{\prime}$ ergibt. Die nahe Übereinstimmung zwischen Beobachtung und Berechnung läßt jedoch mit läucksicht auf das früher für die Form $e$ Gesagte keinen Schluß für die Existenz dieser Form $\{13.1 .1$ z̈ $\}$ zu, da bei so hohen Indices eine Annüherung der Beobachtung an irgend eine Form stets wahrscheinlich ist.

An das zweite lehnl sich ein drittes schmales Individuum an, welches mit II nach dem gewöhnlichen Zwillingsgesetze verwachsen ist. Im Querschnitte (Fig. 14 j trelen dessen Flïchen $p_{\text {III }}$ hervor. An die nach I liegende Flïche $p_{\mathrm{III}}$ schließl sich beiderseits (in Fig. 14 nicht angedeutet) je eine Flïche $\rho$. Da diese letztere Form bei den einfachen Krystallen nur auf der oberen Seite beobachtel wird, und da ferner bei den Zwillingen nach dem vorliegenden gewöhnlichen (iesetze stels die negative Sphenoidfläche des einen Individuums mit der positiven des anderen zur Berührung kommt, so künnen die beiden hervorragenden Sphenoidflächen des dritten Individuums weder den Flächen $a$, noch den Flächen $p^{\prime}$, sondern nur den oberen positiven Flächen $p$ entsprechen. Die Kante $x: x$ des dritten Individuums läge also senkrecht zur Kante $\varkappa_{\mathrm{II}}: \varkappa_{\mathrm{II}}$, genau so wie bei den von Wadia beschriebenen Drillingen. Das gewöhnliche $Z$ willingsgesetz des Kupferkieses muls demnach streng genommen folgende Fassung haben:

Die gegenseitige $Z$ willingslage der beiden Individuen erhält man, indem man beide spiegelbildlich zu einander in bezug auf

Groth, Zeitachrift f. Krystallogr. XlII. 
eine untere positive Sphenoidfläche $(\pi)$ an einander legt und darauf das eine der beiden um $90^{\circ}$ um die Normale zur Basis dreht.

Am Grunde des inneren Winkels des Zwillingsstockes liegt eine weitere Fläche $p_{\mathrm{IV}}$, welche auf die Existenz eines vierten Individuums hinweist,

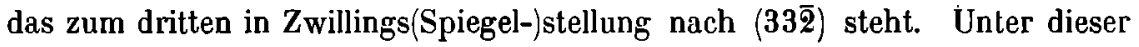
Annahme beträgt der Winkel $p_{\text {IV }}: p_{\text {III }} 5107^{\prime}$; beobachtet wurde $5108^{\prime}$.

Dieses vierte Individuum verhält sich zum ersten fast ebenso, wie das dritte zum zweiten. Stände I : IV in Zwillingsstellung nach dem gewöhn-

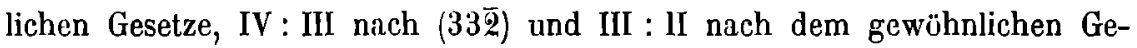
sètze, dann berechnete sich der Normalenwinkel von $c_{\mathrm{I}}: c_{\mathrm{II}}=2\left(\mathbf{3 5}^{\circ} \mathbf{4 0}^{\prime}+\right.$

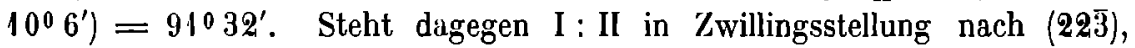
dann berechnet sich der Normalenwinkel $c_{\mathrm{I}}: c_{\mathrm{II}}=2\left(35^{0} 40^{\prime}+11^{0} 27^{\prime}\right)=$ 94.014 '. Beobachtet wurde $c_{\mathrm{I}}: c_{\mathrm{II}}=95^{\circ} 55^{\prime}$.

Derselbe $Z$ willingsstock trägt noch einen weiteren Krystall am Ende des zweiten Individuums (Fig. 13), einen sehr gut ausgebildeten Pyrit. Letzterer ist so orientiert, da $\beta$ seine beiden Flichen $\varepsilon(102)$ und (T02) fast genau, bis auf $2^{0}$, mit je einer Fläche $e$ des Chalkopyrits einspiegeln, und daß die Kante von $\varepsilon: \varepsilon$ des Pyrits beinahe die Fortsetzung der Kante $e_{\mathrm{II}}: e_{\mathrm{II}}$ des Chalkopyrits darstellt. Beim Pyrit mißt $\varepsilon: \varepsilon 53^{0} 8^{\prime}$, beim Chalkopyrit $\left(e\right.$ als $\{15.1 .15\}$ gedeutet) $e: e 55^{0} 7^{\prime}$. Wenn auch ein genaues Zusammenfallen zwischen keiner Fläche des Pyrits und des Chalkopyrits zu erkennen ist, so deutet doch dic erwähnte Orienticrung auf eine gegenseitige Beeinflussung.

Schief durch den ganzen Chalkopyritstock verläuft eine unregelmäßige Absonderung; über der einen Hälfte hat bis zur Absonderungslinie, anscheinend nachträglich, eine parallele Fortwachsung stattgefunden.

\section{II. Über die Structur des Chalkopyrits im allgemeinen.}

1. Über die Unterscheidung der positiven und negativen Formen.

Nach Sadebeck 1 ) ist das positive Sphenoid meist vorwaltend und gestreift, das negative glatt; die Skalenoëder $y\{313\}$ und $s\{513\}$ sollen nur über dem positiven Sphenoid, über dem negativen Sphenoid sollen keine Skalenoëder vorkommen. "Die Skalenoëder sind es daher, wie Sadebeck hervorhebt, welche, wenn sie sich zeigen, die Hemiëdrie erkennen lassen; sie bezeichnen das Sphenoid erster Stellung, nur in einer Stellung auftretend. *

W. Schimper ${ }^{2}$ ) bemerkt hierüber: "Der Kupferkies zeigt eine etwas größere Constanz in der Verschiedenheil der beiden häufigsten Sphenoide, als z. B. die Zinkblende in derjenigen der Tetraëder; schließen wir uns

1) Zeitschr. d. d. geol. Ges. 1868, 20, 395.

z) P. Gr oth, Die Mineraliensammlung der liniverisität Straßburg 1878, S. 54. 
der von Herrn Sadebeck getroflenen Wahl an, so ist dás ersto (positive) Sphenoëder $\{111\}$ stets weniger glănzend, oft ganz matt, mit Eisenoxydhydrat überzogen und stark gestreift nach der Höhenlinie seiner Flächen, also durch Flächen eines Skalenoëders in der Zone (111):(201); dagegen ist das negative Sphenoëder ( $\mid \bar{T} 1)$ meist kleiner ausgebildet, stets glänzend, niemals oxydiert und nicht gestreift. "Dagegen fand Schimper, "daß die beiden von Sadebeck aufgestellten Regeln »die Skalenoëder bezeichnen das positive Sphenoëder", und sdie Skalenoëder treten nur in einer Stellung auf ", unrichtig sind ", indem er außer $\{913\}$ zwei Skalenoëder in positiver und drei in negativer Stellung fand.

Zoltán Toborffy ${ }^{1}$ ) bemerkt bezüglich des Kupferkieses von Pulacaya: "Das Sphenoid $p=x\{111\}$ ist gewöhnlich sehr gerieft, bei einfachen Krystallen nur horizontal, während bei Zwillingen auch eine den Combinationskanten $(111):(101)$ und $(1 T 1):(101)$ parallele Riefung hinzutritt, was triangulare Zeichnungen verursacht. " Das negative Sphenoid $p^{\prime}=$ $*\{1 T 1\}$ hat immer glänzende, fehlerlose Flächen und ist, wenn es mit den positiven zugleich auftritt, immer kleiner als jenes." "Sadebeck und Schimper glaublen zwar an dem glünzenden oder gerieften $\ddot{\Lambda}$ ußeren der Sphenoidflächen den negativen resp. positiven Charakter zu erkennen; manchmal erreichten sie dadurch ihr Ziel, oft waren aber $x\{111\}$ und $\approx\{1 T 1\}$ in physikalischer Hinsicht vollkommen gleich, wodurch eine Orientierung unmöglich wurde. So beschreibt Sadebeck selbst 1866 vom Stahlberge bei Müsen solche Krystalle in einer Weise, welche seiner spïteren Angabe widerspricht. *

Zoltín Toborffy verwendet daher Lösungsmittel zur Unterscheidung und findet, $\operatorname{da} \beta p^{\prime}=\varkappa\{1 T 1\}$ gegen Süuren entschieden widerstandsfähiger ist als die anderen Flächen.

Die tetragonale Grundpyramide erster Art zerfällt bei den vorhin heschriebenen Krystallen von Arakawa nicht nur in positive und negative, sondern auch in obere und untere, im ganzen also in vier Flächengruppen. Bezeichnen wir, wie vorhin, die oberen positiven Flächen mit $p$, die oberen negativen mit $p^{\prime}$ und die unteren positiven mit $\pi\left(\pi^{\prime}, \mathrm{d}\right.$. h. die untere negative Form wurde als Krystalllläche nicht beobachtet), so unterscheiden sich $p$ und $\pi$ einerseits von $p^{\prime}$, andererseits im allgemeinen durch eine größere Neigung zur Streifenbildung. Aber während $p^{\prime}$, wie oben gezeigt wurde, auch eine horizontale Streifung besitzen kann, ist andererseits auch $\pi$ ganz glatt, oder nur mit vereinzelten horizontalen Streifen versehen. Die triangulare Streifung auf $p$ und $\pi$ beschränkt sich keineswegs auf Zwillingskrystalle. Im allgemeinen sind übrigens $p, p^{\prime}$ und $\pi$ weniger gestreift als die meisten anderen Formen.

1) Diese Zeitschr. 1904, 39, 366 . 
Bezüglich der Größe ist zu bemerken, daß $\pi>p$ und $p^{\prime}$ meist kleiner als $\pi$ und $p$.

Sicherer als durch die Streifung und die relative GröBe lassen sich $p^{\prime}$ und $p$ bezüglich $p^{\prime}$ und $\pi$ dadurch von einander unterscheiden, daB (wenigstens bei den Krystallen von Arakawa, wahrscheinlich aber allgemein) die Flächen $m$ und $e$ mit $\pi$ und $p$, nicht aber mit $p^{\prime}$ durch Vicinalflächen verknüp ft sind, so daB $m$ und $e$ scheinbar stets näher bei $\pi$ und $p$ als bei $p^{\prime}$ liegen.

\section{2. Über die dem Chalkopyrit zukommende Symmetrieklasse.}

Bekanntlich wurde der Kupferkies vor Haidinger für regulär gehalten. Mit Hilfe des Reflexionsgoniometers erkannte dieser 1822, da $ß$ die Kanten des scheinbar regulïren Oktaëders beim Kupferkies teils größer $\left(109^{0} 53^{\prime}\right)$, teils kleiner $\left(108^{0} 40^{\prime}\right)$ sind, als bei der regulären Form $\left(109^{n} 28^{\prime}\right)$. Der Kupferkies gilt seitdem allgemein als tetragonal und sein Axenverhältnis ist nach II aidinger: $a: c=1: 0,98525$.

Der Unterschied der Flächenausbildung zwischen den abwechselnden Oktanten brachte dann den Chalkopyrit in die didigonal-skalenoëdrische Klasse. Die Formen dieser Klasse besitzen zwei zu einander senkrechte und mit einander gleichwertige zweizühlige Deckaxen $\left(2 A_{1}{ }^{2}\right.$, welche den Kanlen $e: c$ parallel gehen, ferner eine ebenfalls zweizählige, aber mit den vorigen ungleichwertige, Deckaxe $\left(A_{3}{ }^{2}\right.$, welche zur Fläche $c$ senkrecht steht, die IIauptaxe, ferner zwei gewöhnliche Spiegelebenen $(2 E)$, parallel zu je einer Kante $p: c$ und der IIauptaxe, endlich eine Drehspiegelebene $\left(P_{3}{ }^{2}\right)$ parallel zur Fläche $c$.

Bei vorstehend beschriebenen Typen Nr. 4 und Nr. 3 der japanischen Kupferkiese fehlen in ganz gesetzmäßiger Weise die Drehspiegelebene und die beiden gleichwertigen Deckaxen; die Krystalle sind hemimorph nach der verticalen Deckaxe. Als Symmetrieelemente sind noch vorhanden die zweizählige verticale Deckaxe $\left(A_{3}{ }^{2}\right)$ und die beiden gleichwertigen Spiegelebenen $(2 E)$. Infolgedessen gehören diese beiden Typen in das rhombische system, und zwar in die rhombisch-pyramidale Klasse.

Es fragt sich: 1) Sind diese beiden Typen nur die Folge einer zufälligen, lediglich durch äußere Verhältnisse bedingten Anomalie?

oder 2) stellen dieselben eine eigene Mineralspecies dar?

oder 3) darf der Chalkopyrit überhaupt nur als rhombisch-hemimorph gelten?

ad 1). Es ist sehr merkwürdig, daß die bisherigen Beobachtungen am Kupferkies niemals Veranlassung gaben, das tetragonale Syslem in Frage 
zu stellen. Der Grund liegt darin, daß eine derartig auffallende hemimorphe Ausbildung beim Kupferkies anderwärts äußerst selten ist. Lewis ${ }^{1}$ erwähnt, daß Messungen zwischen tautozonalen Flächen auf gegenüberliegenden Seiten der Hauptaxe oft eine $A$ bweichung von $4^{0}$ bis $5^{0}$ ergeben, und daß sich gelegentlich feststellen ließ, daß auf der einen Seite die Fläche (441) oder (551) stark ausgebildet war, während auf der anderen Seite die Fläche ( $\bar{\top} \bar{\top} 0$ ) stark hervortrat. Auf einer Kupferkiesstufe von Cornwallis beobachtete ich auch einen Krystall, welcher genau dem vierten Typus von Wada entspricht. Indessen sind derarlige Beobachtungen doch nur Seltenheiten.

Trolzdem ist die Annahme, daß die geringere Symmetrie der beschriebenen japanischen und der anderwärts nur vereinzelt vorkommenden Krystalle nur zufälig sei, ausgeschlossen.

Wada erwähnt, daß diese Form in Japan »kein seltener 'Typus sei . Sie wurde zuerst 1892 und dann später von Wakabayashi in großer Menge ("large quantity ") gesammelt. Mir lagen, wie erwähnt, annühernd 50 Exemplare dieser Art vor. Die Beschrünkung von $e$ und $p^{\prime}$ auf die obere Hälfte, die völlige Verschiedenheit und Unabhängigkeit von $p$ und $\pi$ ist bei allen Exemplaren so auffallend, daß sich eine structurelle Verschiedenheit zwischen der oberen und der unteren Hälfte nicht in $\Lambda$ brede stellen läßt.

ad 2). Daß aber andererseits der vierte und fünfte Typus eine eigene, von den übrigen verschiedene, Mineralspecies darstellen, ist ebenfalls ausgeschlossen.

Als specifisches Gewicht für die Krystalle des vierten bezüglich fünften Typus ergab die Untersuchung den Wert 4,139; für Kupferkies wird im allgemeinen der Wert 4,1 bis 4,3 angegeben.

Als Kante (001): (111) wurde für jene der Mittelwert $54023^{\prime}$ gefunden, für die gewöhnliche Ausbildung fand Haidinger $\mathbf{5 4} \mathbf{2 0}^{\prime}$.

Auch dic besonderen Ligentümlichkeiten der Flächen der oberen vier Oktanten der hemimorphen Krystalle von Arakawa entsprechen den Beobachtungen Penfields an skalenö̈drisch erscheinenden Krystallen aus den French Creek-Eisengruben.

ad 3). Wenn nun die genannten japanischen Typen weder eine zufällige Anomalie, noch cine eigene Species darstellen, dann kann der Kupferkies überhaupt nicht mehr als didigonal-skillenoëdrisch gelten, sondern nur noch als rhombisch-pyramidal. Die an beiden Enden der verticalen Axe gleichartig ausgebildeten Krystalle müssen dann in der Regel wohl als Zwillinge aufgefaßt werden, für welche die den rhombisch-pyramidalen Formen fehlenden Symmetrieelemente der didigonal-skalenoëdrischen Klasse

1; Diese \%eitschr. 34, 322. 
als Zwillingselemente vorhanden sind. In gewissen Fällen kann aber auch durch einfache Combination von oberen und unteren Flächen anscheinend holomorphe Ausbildung zustande kommen.

Die Kupferkieskrystalle der gewöhnlichen Ausbildungsweise sind also in der Regel Zwillinge nach dem Gesetze: Zwillingsaxe ist die bei der gewöhnlichen Aufstellung als Nebenaxe bezeichnete Kante $c: e$.

Bei den beiden oben beschriebenen rechteckigen, nach $c$ tafelförmigen Krystallen scheint auch die dort erwähnte Naht auf eine Zwillingsgrenze zu deuten. Sowohl bei diesen Platten, als bei den von Wada beschriebenen Drillingen und dem von mir beschriebenen Vierling, als auch bei den von Penfield beschriebenen, scheinbar holomorphen Krystallen ist die obere Hälfte nach außen gerichtet, so daß die Flächen $\pi$, d. h. das flächenärmere Ende, teilweise oder völlig von der Oberfläche verschwinden.

Die Fig. 9, 10, 11 und 12 geben die Krystalle in rhombischer Aufstellung. Das Axenverhältnis wird nunmehr

$$
a: b: c=0,7071: 0,7071: 0,9852=1: 1: 1,3933 \text {. }
$$

Sind die Parameter einer Fläche des Kupferkieses in der gewöhnlichen tetragonalen Darstellung $a: n a: m c$, so sind dieselben in Bezug auf vorstehendes rhombisches Verhältnis

$$
\lambda a^{\prime}: \nu b^{\prime}: \mu c^{\prime}, \quad \text { wobei } \quad \lambda=\frac{2 n}{n-1}, \quad \nu=\frac{2 n}{n+1}, \quad \mu=m .
$$

Hiernach ergibt sich nachstehende Übersicht der einzelnen im vorhergehenden erwähnten Formen in tetragonaler und in rhombischer Deutung:

$\begin{array}{cccccc}\text { Zeichen: } & \text { Tetragonal: } & \text { Rhombisch: } & \text { Zeichen: } & \text { Tetragonal: } & \text { Rhombisch: } \\ p & \{111\} & \{011\} & n^{\prime} & \{1 T 2\} & \{102\} \\ p^{\prime} & \{1 T 1\} & \{101\} & \gamma^{\prime} & \{1 \bar{T}\} & \{105\} \\ \pi & \{1 \overline{1}\} & \{10 \bar{T}\} & \mu & \{205\} & \{115\} \\ e & \{101\} & \{112\} & x & \{113\} & \{013\} \\ c & \{001\} & \{001\} & & \{22 \overline{3}\} & \{02 \overline{3}\} \\ m & \{1 \overline{1} 0\} & \{100\} & r & \{33 \overline{2}\} & \{03 \overline{2}\} \\ n & \{112\} & \{012\} & \approx & \{201\} & \{111\}\end{array}$

3. Über die pseudokubische Natur des Chalkopyrits.

V. Goldschmidt (»Krystallographische Winkeltabellen«) und E. v. Fedorow ${ }^{1)}$ haben auf Grund der Complicationsregel dem Chalkopyrit eine von der gewöhnlichen abweichende tetragonale Aufstellung gegeben, deren Orientierung und Axenverhältnis genau mit der vorigen, aber infolge der rhombischen Symmetrie notwendig gewordenen Axenwahl, übereinstimmt.

1) Diese Zeitschr. 35, 57 und 37, з9. 
Da hierbei die pseudokubische Natur, welche bei der gewöhnlichen Aufstellung des Chalkopyrits mit dem Axenverhältnisse $a: c=1: 0,9852$ so auffallend hervortritt, sich weniger bemerkbar macht, so äußerte Fedorow die Vermutung, "daß der pseudokubische Habitus der ("Kupferkies «-) Krystalle so zu sagen zufällig sei« $\left.{ }^{1}\right)$.

Für die pseudokubische Natur des Kupferkieses sprechen:

1) die hervorragende Bedeutung der dem regulären Oktaëder äußerst nahe kommenden Formen $p, p^{\prime}$ und $\pi$;

2) der häufige pseudotrigonale, oder auch pseudoreguläre Habitus;

3) die parallelen Verwachsungen und Zwillingsbildungen.

ad 1). Die Sphenoide $p$ und $p^{\prime}$ bezüglich $\pi$ treten nicht nur häufig selbständig beim Kupferkies auf, sondern sie kommen in alternierender Ausbildung mit fast allen anderen Flächen vor, so daß diese nach ihren Combinationskanten mit jenen gestreift sind. Mehrfach gewinnt man den Eindruck, daß alle anderen Flächen nur die Böschungen zwischen den verschiedenen, von den Sphenoiden gebildeten Terrassen darstellen 2); dic Krystalle erscheinen geschichtet nach den Flächen des posiliven oder negativen Grundsphenoids, alle anderen Formen werden von den Schichtendigungen gebildet.

ad 2). Die tetragonale Symmetrie unterscheidet sich von der regulären durch den Mangel der trigonalen Axe. Nun ist aber der Ilabitus der Kupferkieskrystalle in vielen Fällen ein derartiger, daß durch die Combinationen die den einfachen Formen fehlende trigonale Symmetrie annähernd hergestellt wird. Ein derartiges Beispiel liefert z. B. die dreiseitige Platte (Fig. 12). Die Flächen $e$ und $m$ entsprechen einander in bezug auf ihre fast gleiche Neigung zu $p^{\prime}$, ihre Abweichung von der normalen Lage und ihre Streifung.

Die von Wada abgebildeten Combinationen von $y\{313\}$ und $X\{331\}$ (Fig. 2), sowie von $\chi\{212\}$ und $t\{221\}$ (Fig. 3) gleichen völlig in bezug auf Winkel und IIabitus den entsprechenden regulären Delloiddodekaëdern. Andere Beispiele dieser Art, trigonal ausgehildete /willinge, erwïhnt Penfield $\left.{ }^{\jmath}\right)$ und bemerkt: „Diese ' $/$ willinge, welche ganz symmetrisch entwickelt sind, scheinen gar nicht selten zu sein; in der Richtung der Zwillingsaxe gesehen, besitzen dieselben hexagonalen Umriß, bestehend aus einem kurzen, hexagonalen Prisma zweiter Ordnung am einen Ende in Combination mit einem einfachen Rhomboëder, im anderen mit zwei Rhomboëdern in Zwillingsstcllung. Diese Anpissung eines tetragonalen hemiëdrischen Minerals an hexagonale Symmetrie ist gewil sehr auflallend und erinnert an die Neigung des Kupfers, sich in derselben Weise zu entwickeln. *

1) Diese Zeilschr. 35, 59.

2) Vgl. ebenda 36, $111 . \quad 3$ E Eubenda 18, 515. 
Die auffallende Ähnlichkeit in der Ausbildungsweise von Kupferkies und Kupfer hat bekanntlich auch schon früher Laspeyres an dem Vorkommen von der Grube Heinrichssegen bei Müsen gezeigt 1).

In Drusenräumen zwischen Quarz und Pyrit fand er sborsten- bis haarfeine, in Form wie in Farbe allerdings etwas an Millerit erinnernde, Krystalle von Kupferkies, teils einzeln, teils büschelförmig oder verfilzt aufgewachsen, zum größten Teile jedoch zu den zierlichsten, bald gitterförmigen, bald sternförmigen, bald federschweifahnlichen und selbst blechförmigen, sogenannten gestrickten Formen stets unter $60^{\circ}$ bezüglich $120^{\circ}$ und immer nur in einer Ebene an einander gefügt«. Auftauchende Bedenken, ob ein tetragonales, wenngleich dem regulären Krystallsysteme geometrisch nahestehendes Mineral die den regulären Metallen zukommende $\Lambda$ ggregatform so täuschend nachahmen könne, veranlaßten Laspeyres zu einer genauen quantitativen Analyse, welche die Formel des Kupferkieses außer Zweifel stellte. „Prismatisch ausgebildete Krystalle von Kupferkies sind bisher noch nicht bekannt geworden, geschweige denn borsten- oder haarförmige. "

Da Messungen nicht möglich waren, so vermutete Laspeyres auf Grund der Analogie mit den gestrickten Formen der regulären Metalle, daß die borsten- und haarfürmigen Krystalle nach der Zonenaxe der Flächen (111), (001), (TTI), (TTT), (00T), (11T) gestreckte Gebilde seien.

Die hier hervorgehobene Lücke, das Fehlen von prismatisch ausgebildeten Kupferkieskrystallen, ist durch die japanischen ausgefüllt. Eine der vermuteten Drillingsbildung der von Laspeyres beschriebenen trigonalen Gebilde entsprechende Bildung isl zwar bei den japanischen Krystallen nicht beobachtet worden, aber die unter dieser Voraussetzung abgeleitete Längsrichtung der haarförmigen Gebilde stimmt genau mit der bei den japanischen Krystallen durch Messung festgeleglen Längsrichtung überein. Während bei den gestrickten l'ormen der regulären Metalle die Unter- und Oberseite zu einander in Zwillingsstellung sich befinden, schloß Laspeyres, daß dies bei den trigonalen Formen des Kupferkieses nicht der Fall sei. Damit steht in Übereinslimmung, daß bei den japanischen dünnplattigen Krystallen die obere und unlere Seite zwar bei den nach (001) tafeligen reckteckigen, nicht aber bei der nach (1 $1 \bar{T}$ ) tafeligen trigonalen Platte in Zwillingsstellung stehen.

ad 3). Im vorstehenden wurde eine Verwachsung von Kupferkies mit Eisenkies beschrieben, bei der nur ein annähernder Parallelismus nachweisbar ist. $0 . M$ ügge beschreibt ${ }^{2}$ ) jedoch auch eine gesetzmüßige Verwachsung von Kupferkies mit Kobaltglanz, bei welcher sämtliche geradzähligen

1) Diese Zeitschr. 20, 529 IT.

2) Jahrb. I. Min. usw., Beil.-Bcl. 16, 3:34; Ref. diese Zeitschr. 41, 628. 
Symmetrieaxen beider Mineralien genau parallel liegen, A. Pelikan ${ }^{1}$ ) eine solche von Kupferkies mit Bleiglanz; Verwachsungen von Kupferkies mit Fahlerz und Zinkblende sind wiederholt beschrieben worden. In allen diesen Fällen findet also gesetzmäßige Verwachsung von Chalkopyrit mit regulären Sulfiden statt, und dabei gehüren letztere sowohl der holoëdrischen, als auch verschiedenen hemiëdrischen Klassen des regulären Systemes an. Der Unterschied der Structur bei den Mineralien der verschiedenen Klassen eines Systems muß also von anderer Art sein, als der Unterschied der verschiedenen Systeme.

Beim Kupferkies werden folgende 7 willingsgesetze angegeben:

1) Zwillingsaxe die Normale zu $p$ (vgl. S. 49),

2) ' ' wwillingsebene $e$,

3) Zwillingsebene $a, b$ oder $c$,

4) Zwillingsaxe die Kante $e: c$ (vgl. S. 54).

Beim regulären Würfel stellen die drei Verbindungen gegenüberliegender Flächenschwerpunkte vierzählige Deckaxen dar, die vier Verbindungen der gegenüberliegenden dreikantigen Ecken dreizühlige, die sechs Verbindungen der gegenüberliegenden Kanten zweizählige Deckaxen. Die vierzähligen Axen stehen senkrecht zu den Würfelfl̈̈chen, die dreizähligen zu den Oktaëderflächen, die zweizähligen zu den Rhombendodekaëderflächen. Als weitere bevorzugte Richtungen können die Höhenlinien der Oktaëderflächen gelten, sie stehen senkrecht zu den Flächen des Ikositetraëders \{112\}, d. h. senkrecht zu einer dreizühligen und zu einer zweizähligen Deckaxe. Da aber erst nach Drehung um $360^{\circ}$ Congruenz eintritt, so können sie nur als einzählige Deckaxen gelten. In der holoëdrischen Klasse des regulären Systems sind die beiden entgegengesetzten Richtungen der mehrzähligen Axen gleichwertig. In einer zu (11I) senkrechten, durch eine der drei Höhenlinien von (111) lindurch geleglen Ebene hat die betreffende Ilühenlinie von (111) und von (TTT) aus gesehen entgegengesetzte Bedeutung $\underset{a b}{\stackrel{b}{a} ;}$; für eine derarlige Erscheinung. habe ich früher den Namen Enantiopolarität vorgeschlagen; er deckt sich in gewissem Sinne mit »centrisch-symmetrisch $₫$.

Beim Chalkopyrit sind alle Richtungen, welche den vier Arten von bevorzugten Richtungen entsprechen, hemimorph mit Ausnahme der vier in der Basisebene liegenden.

Beim Spinellgesetze aller Klassen des regulären Systems liegen die drei der Zwillingsebene parallelen einzïhligen $A x e n$ zu beiden Seiten der Zwillingsgrenze parallel, aber der Richtungssinn der enantiopolaren (einzähligen) Axen ist durch die $Z$ willingsstellung umgekehrt worden. Dies gilt sowohl

1 'Tschermaks mineral. u. petrogyr. Milt. 16, s8; Ref. diese \%eilschr. 30, s11. 
für die Definition: Spiegelebene ist (111), als für: Drehaxe ist die Normale zu (111). Auch für das erste Zwillingsgesetz des Chalkopyrits gilt diese Beziehung.

Durch das zweite Zwillingsgesetz des Chalkopyrits werden die in der Zwillingsebene liegenden, den zweizähligen und den vierzähligen Axen entsprechenden Richtungen umgekehrt.

Durch das dritte $Z$ willingsgesetz in der Fassung: Zwillingsebene ist die Ebene $a$ oder $b$ werden die den dreizähligen Axen, in der Fassung: Zwillingsebene ist die Fläche $c$ sowohl die den dreizähligen, als auch die den vierzähligen $\Lambda$ xen entsprechenden Richtungen entgegengesetzt gerichtet.

Durch das vierte Gesetz wird die der vierzähligen verticalen Axe entsprechende Richtung umgekehrt.

Sämtliche Zwillingsgesetze des Kupferkieses haben somit den Erfolg, daß hemimorphe bevorzugte Richtungen bei beiden Individuen zwar parallel bleiben, aber entgegengesetzt gerichtet werden, da $\beta$ also das $Z$ willingsaggregat die den Einzelindividuen fehlenden, aber den regulär holoëdrischen Körpern zukommenden Symmetrieeigenschaften durch die Zwillingselemente ersetzt.

F. Wallerant hat bereits früher ${ }^{1}$ ) darauf hingewiesen, daß bei den Verwachsungen von Chalkopyrit mit Tetraëdrit, Sphalerit, Cobaltit und Polybasit die bevorzugten Richtungen bei beiden mit einander verwachsenen Mineralien parallel seien. Zur Erklärung dieser Erscheinung nahm er an, daß die Partikeln nach diesen Richtungen centrale Kraftwirkung ausübten. Nach der von mir aufgestellten Theorie der stehenden Wellen ${ }^{2}$ ) erklärt sich die Erscheinung sowohl hei den Zwillingen, als bei den parallelen Verwachsungen dadurch, daß auf den bevorzugten Geraden Schwingungen stehender Wellen stattfinden, und daß deren Knotenpunkte auch bei an sich hemimorphen Richtungen in der parallelen und in der Zwillingsstellung die gleiche Lage haben.

\section{4. Über eine hypothetische Molekularstructur des Chalkopyrits.}

Die rhombische Symmelrie des Kupferkieses bestimmt unabhängig von Complication, Spaltung und Pseudosymmetrie nicht dic Fläche $p$, sondern $\approx$ zur Grundform. Die Complicationsregel und die Spaltung nach $\approx$ führen unabhängig hiervon für sich zur gleichen Grundform. Jede Structurhypothese muß aber trotzdem auler der rhombischen Symmetrie auch die ebenso sicher stehende pseudokubische Natur des Chalkopyrits zum Ausdrucke bringen.

1) Bull. d. I. soc. fr. d. min. 1902, 25, 180 fr.; Ref. diese Zeitschr. 39, 205.

2) Vgl. diese Zeilschr. 42, 466 u. 1. 
Der Umstand, daß beim Kupferkies fast stels Zwillinge nach dem vierten Gesetze vorliegen, läßt vermuten, daß hier eine Molekulargruppe besteht, welche mit Bezug auf die $\Lambda$ nordnung der Schwerpunkte der die Gruppe zusammensetzenden Einzelmoleküle die Symmetrie der didigonalskalenoëdrischen Klasse besitzt, der aber mit Rücksicht auf die Eigenschaften der einzelnen Moleküle nur eine geringere Symmetrie zukommt. Eine derartige Gruppe stellt z. B. Fig. 15 dar. Die Schwerpunkte der vier Moleküle mögen die Ecken eines tetragonalen Sphenoids bilden. Mit Rücksicht auf die durch die Pfeile angedeuteten, jedes Einzelmolekül umkreisenden Bewegungen künnen die Moleküle aber sämtlich nach den Schnittlinien der Bahnen hemimorph sein. Liegt z. B. bei jedem der vier Moleküle der gemeinsame Brennpunkt der elliptisch gedachten Bahnen oberhalb des Mittelpunktes derjenigen Ellipse, deren längere Axe vertical steht, dann ist die Gruppe nur rhombisch und nach der Verticalen hemimorph.

Durch Drehung der Gruppe um eine durch ihren Schwerpunkt von vorn nach hinten, oder von rechts nach links gehende horizontale Axe ( $a$ oder $b$ ) um $180^{\circ}$ bleibt die Verteilung der Schwerpunkte der vier Moleküle im Raume congruent, aber die Richtung der hemimorphen verticalen Axe wird umgekehrt.

Die Individualität einer derartigen Gruppe wäre nach einer früher ${ }^{1}$ ) von mir gemachten $A$ nnahme dadurch bedingt, daß alle gleichnamigen (hier Süd-)Pole der Bahnen nach außen gerichtet sind.

Wiederholt sich die Gruppe im Raume derartig, daß die Gruppenschwerpunkte quadratische Prismen bilden (Fig. 16), dann bilden die Schwerpunkte der Einzelmoleküle ein Bravaissches Gitter nach einem quadratischen Prisma mit einem Massenpunkte in der Mitte. Die mit vollen Linien angedeuteten Ellipsen 1 und 2 liegen in der Zeichnungsebene, die gestrichelten in einer zu diesen parallelen, aber im Alsstande der halben $c$-Axe tiefer gelegenen Ebene.

Dasselbe Gitter kann aber auch als ein in der lichtung der verticalen Axe nur wenig deformiertes (hier comprimiertes) reguläres Gitter nach dem Würfel mit einem Massenpunkte in der Mitte der Würfelliüchen angesehen werden. Bei Fig. 17 stehen die Punkte an Stelle der Ellipsen 1 und 2, die kleinen Ringe an Stelle der Ellipsen 3 und 4 .

Mit Rücksicht auf die Bewegungsrichtungen aber muß dieses Gitter im Sohnckeschen Sinne als cine Durchdringung von vier annähernd kubischen einfachen (iittern angeschen werden.

Liegen die (tangentialen) Hauplwachstumsrichtungen innerhalb der Gruppe in den Verbindungslinien der Finzelmoleküle, so bestehen dieselben

1) Vgl. diese Zeitschr. 36, 887 
60 J. Beckenkamp. Über Chalkopyrit von Arakawa, Provinz Ugo-in Japan, usw.

entsprechend dem rhombischen Systeme, bei welchem die zur Zeichnungsebene senkrechten, durch 1,2 und durch 3,4 gehenden Ebenen Spiegelebenen sind, aus ungleichwertigen Richtungen und zwar sind dies:

1) Die Verbindungslinien der Moleküle 1 und 2,
2)

Jedoch begünstigt das Bestreben nach gleichmäßiger Abrundung in der Regel wenigstens eine einmalige Zwillingsbildung nach der Kante $e: c$, so daß dann die Richtung 1:2 der unteren Hälfte des Krystalles in die Richtung $3: 4$ der oberen Hälfte füllt, und beide Richtungen einander gleichwertig erscheinen. 